\title{
An Analytical Model for a Sectored Cellular Network with Embedded Small Cells
}

\author{
Tsang-Ling Sheu ${ }^{1}$, Yan-Jing $\mathrm{Wu}^{2}$, Yi-Hsun Lin ${ }^{1}$ \\ ${ }^{1}$ Department of Electrical Engineering, National Sun Yat-sen University, Kaohsiung \\ ${ }^{2}$ Department of Information Technology and Communication, Shih Chien University, Kaohsiung Campus, Kaohsiung \\ Email: *sheu@ee.nsysu.edu.tw, yanjing@g2.usc.du.tw, lue800205@atm.ee.nsysu.edu.tw
}

How to cite this paper: Sheu, T.-L., $\mathrm{Wu}$, Y.-J. and Lin, Y.-H. (2021) An Analytical Model for a Sectored Cellular Network with Embedded Small Cells. Journal of Computer and Communications, 9, 128-149. https://doi.org/10.4236/jcc.2021.911009

Received: October 14, 2021

Accepted: November 22, 2021

Published: November 25, 2021

Copyright $\odot 2021$ by author(s) and Scientific Research Publishing Inc. This work is licensed under the Creative Commons Attribution International License (CC BY 4.0).

http://creativecommons.org/licenses/by/4.0/ (c) (i) Open Access

\begin{abstract}
In order to handle the huge amounts of data transmitted through hot spots and to increase cell coverage in buildings, this paper proposes a sectored cellular network with small cells (SCNSC) embedded in each sector. Two region types, sectors and variable-radius small cells are classified in an SCNSC macro cell. Because the system capacity is enhanced by using fractional frequency reuse (FFR), the overall channel bandwidth is divided into two or three frequency bands depending on whether the number of sectors in a macro cell is even or odd. For the purpose of topological evaluation on the proposed SCNSC, we build an analytical model using Markov chain theory. The performance measures include new-call blocking and handoff-call dropping probabilities, average waiting times, and normalized throughputs in each sector and its embedded small cell by varying mobile stations' moving speeds, the number of sectors, and the radius size of an embedded small cell. From the evaluation results, it is demonstrated that adjusting either the number of sectors or the coverage of an embedded cell can satisfy different service demands.
\end{abstract}

\section{Keywords}

Sectored Cell, Small Cell, New-Call Blocking, Handoff-Call Dropping, Markov Chain

\section{Introduction}

Advancements in mobile wireless communication technology have led to a tremendous growth in the number of wireless devices and the volume of data transmitted, respectively. One of the primary goals for fifth generation (5G) communication systems is to provide up to 1 Gbps data rate anywhere and anytime. 
This can be achieved by combining several novel communication technologies, such as massive multiple-input multiple-output (MIMO), millimeter wave (mmwave), and small cell deployment [1] [2]. In recent decades, several schemes [3] [4] [5] have been presented to alleviate the problem of high link interruption probability due to insufficient radio resource by delaying or advancing the instant at the beginning of handoff procedure. On the other hand, the authors in [6] [7] [8] [9] focused on selecting the target base station (BS) for a mobile station (MS) to reduce handoff times based on certain criteria related to MS signal strength. Further, several works [10] [11] [12] intended to lower link interruption rate with their proposed channel allocation mechanisms.

However, the previously-proposed research works have not considered the topology of a sectored macro cell with small cells. To enhance cell coverage and data rate for a large number of users, the coverage performance of small cells has been well studied. Multiple classes of BSs, macro BSs, hotspot BSs, and femtocell BSs, are installed in a heterogeneous cellular network. On the other hand, sectored antennas can be deployed to support fractional frequency reuse (FFR) in a cell to increase subscriber capacity [13]. The works in the literature [14]-[27] are divided into three categories as below.

1) A macro cell using an omnidirectional antenna is installed with multiple small cells, which are referred to as either femto cells or pico cells [14] [15] [16] [17].

2) A macro cell using a multi-directional antenna consists of multiple sectors [18] [19] [20] [21].

3) A macro cell using a multi-directional antenna consists of multiple sectors and one or more small cells are installed in each sector [22]-[27].

As to the third category, Wu et al. [22] [23] presented a three-sectored macro cell architecture with a femtocell inside each sector and a femtocell exclusion region (FER) around a macro BS. The expression of FER radius as a function of the azimuth angle relative to the macro BS was derived, which can be directly used to divide the macro cell coverage area into inner and outer regions for cochannel assignments to the macro cell and femtocells. Further from the proceeding works, Kalbkhani et al. [24] divided a macro cell into six sectors with multiple femtocells randomly installed in some sectors. According to the amount of received interferences, they presented an algorithm for channel allocation in femtocells and a closed-form formula for femtocell downlink outage probability. Tsai [25] proposed that installing three-dimension directional antennas on small cells can mitigate two-tier interference of the ultra-dense heterogeneous smallcell network and investigated the impacts of the three-dimension directional antennas and the resource block usage ratio on the system capacity and link reliability in an apartment building. Dividing a macro cell into a center zone and an edge zone (the cell coverage beyond the center zone) of three sectors, the authors in [26] aimed to mitigate interference between a macro cell and multiple femtocells in the center and edge zones by using fractional frequency reuse (FFR) under the macro cell allocating a frequency band and the femtocells selecting the 
sub-bands.

Although the aforementioned schemes [22] [23] [24] [25] [26] have improved the outage probability, including the new-call blocking and handoff-call dropping probabilities, in a macro cell with multiple small cells, they have not considered the influences of MS mobility, the coverage size of a small cell, and the number of sectors on the outage probability of a call moving from adjacent cells. Our interest in this paper is to design a sectored cellular network with small cells (SCNSC) embedded in each sector. Two region types, sectors and variable-radius small cells are classified in an SCNSC macro cell. The proposed SCNSC can increase the system capacity by using fractional frequency reuse (FFR) since two sectors that are not adjacent can allocate the same frequency band. Each frequency band is further divided into two portions assigned to sectors and small cells, respectively. For performance evaluation, we derive the new-call blocking and handoff-call dropping probabilities, average waiting times, and normalized throughputs in each sector and its embedded small cell in terms of the call generation rate, MS moving speed, the number of sectors, and the radius size of a small cell using Markov chain theory.

The remainder of this paper is organized as follows. Section 2 defines the proposed SCNSC. In Section 3, we build an analytical model for the SCNSC by using a multi-dimensional Markov chain. The analytical results and discussions are presented in Section 4. Finally, concluding remarks are given in Section 5.

\section{The Proposed SCNSC}

\subsection{System Model}

As illustrated in Figure 1, a macro cell in the proposed SCNSC consists of two region types, sectors and variable-radius small cells. The coverage of a macro cell is sectored by using a multi-directional antenna and a variable-radius small cell is installed within each sector. Assuming that the maximum coverage of a small cell is an inscribed circle within a sector, a small cell coverage spanning the boundary of two sectors is not allowable.

We summarize the symbols and denotations for the SCNSC in Table 1. Let $n$ be the number of sectors for a macro cell. $R$ and $r$, respectively, represent the radiuses of a macro cell and a small cell. $A r$ is defined as the ratio of a small cell coverage to a sector region, as expressed in Equation (1). The system capacity is enhanced by using FFR. Therefore, the overall channel bandwidth is divided into $n_{f}$ frequency bands, where $n_{f}$ is two (three) if $n$ is even (odd). Figure 2 shows channel allocation for the system when $n$ is even. As shown in Figure 2(a), the system bandwidth is divided into two frequency bands in different colors and each frequency band is composed of two portions, one is for small cells and the other is for sectors. As illustrated in Figure 2(b), in order to reduce co-channel interference, two adjacent sectors allocate different frequency bands and the two embedded small cells use different frequency bands from their respective sectors. Similarly, Figure 3 shows channel allocation for the system when $\mathrm{n}$ is odd. Let $N$ 


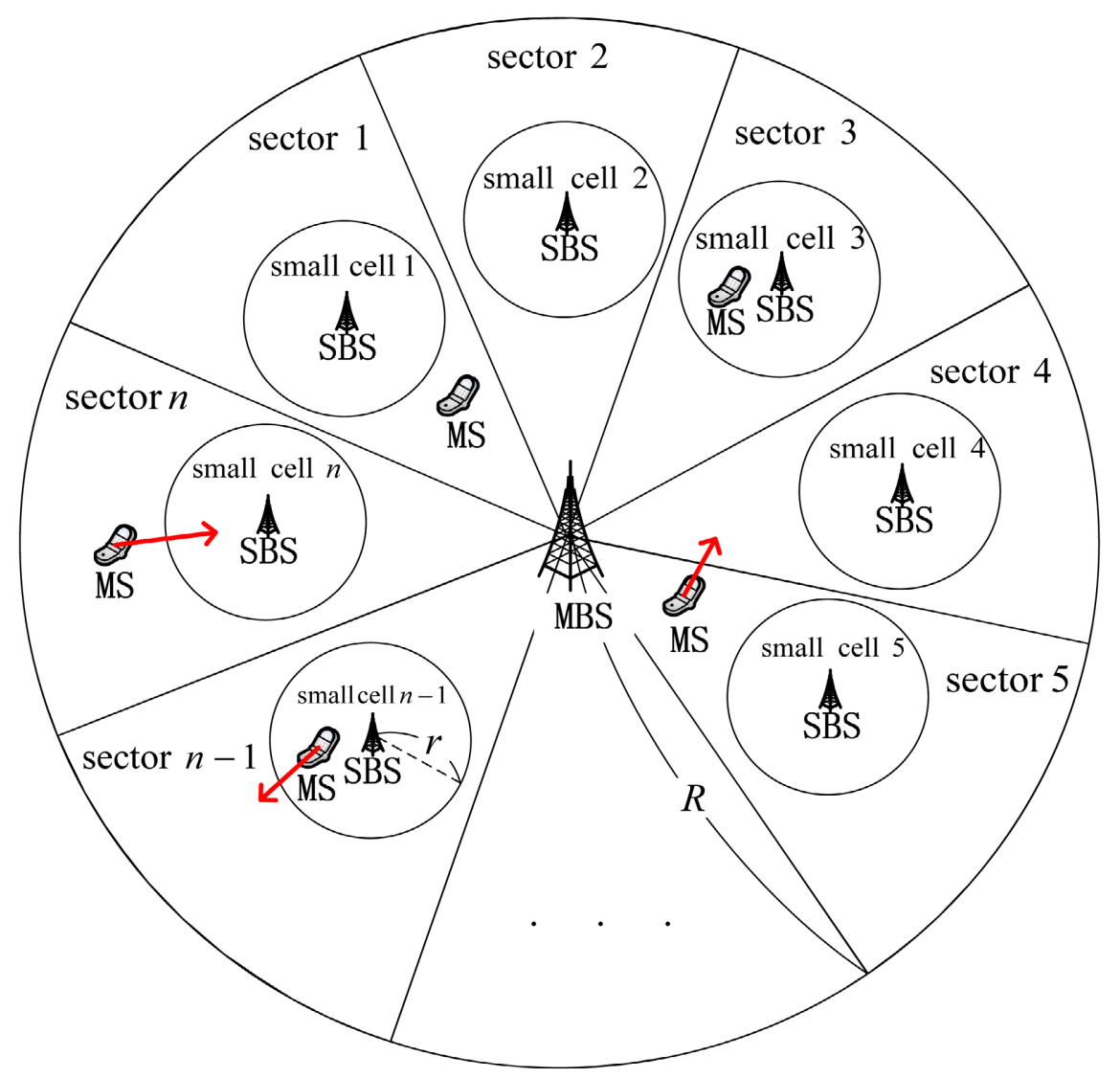

Figure 1. System architecture for the SCNSC.

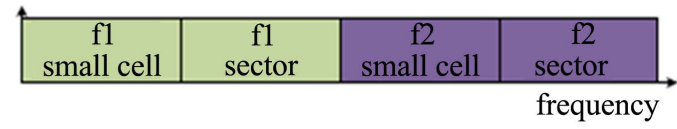

(a)

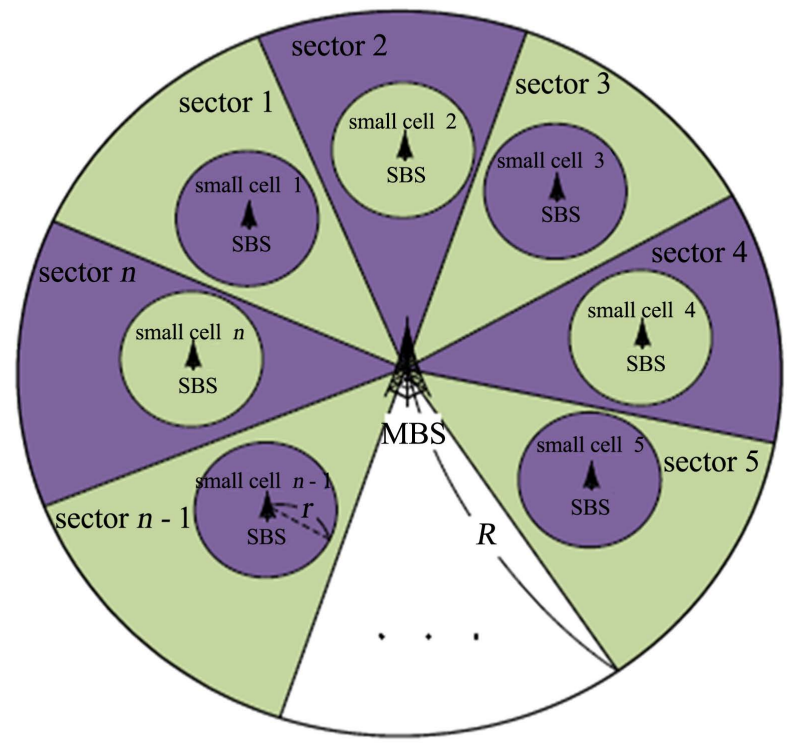

(b)

Figure 2. Channel allocation as $n$ is even. (a) $n_{f}=2$; (b) Frequency utilization map. 


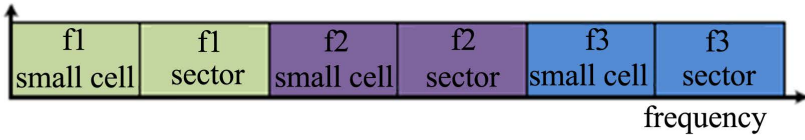

(a)

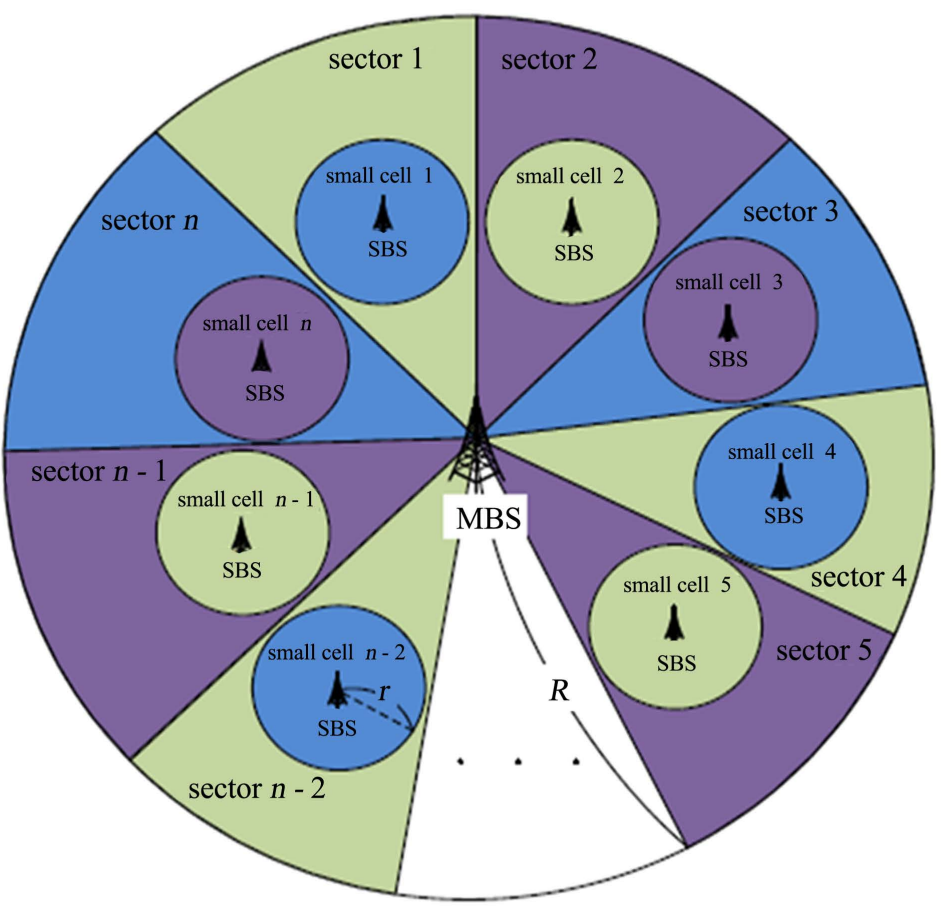

(b)

Figure 3. Channel allocation as $n$ is odd. (a) $n_{f}=3$; (b) Frequency utilization map.

Table 1. Symbols and denotations of the SCNSC.

\begin{tabular}{|c|c|}
\hline Symbols & Denotations \\
\hline$n$ & the number of sectors for a macro cell \\
\hline$R$ & the radius of a macro cell (in the unit meters) \\
\hline$r$ & the radius of a small cell (in the unit meters) \\
\hline$A r$ & a small cell coverage divided by to a sector region \\
\hline$n_{f}$ & the number of frequency bands (either 2 or 3 ) \\
\hline$N$ & the total number of channels in the system \\
\hline$N_{s r}$ & the total number of available channels for a sector \\
\hline$N_{s c}$ & the total number of available channels for a small cell \\
\hline$N C_{s r}$ & new calls initiating in a sector region \\
\hline$N C_{s c}$ & new calls initiating in a small cell coverage \\
\hline$H C_{s r_{-} s r}$ & handoff calls of MSs moving between two sectors \\
\hline$H C_{s L_{-} s c}$ & handoff calls of MSs moving from a sector to its embedded small cell \\
\hline$H C_{s c_{s} s r}$ & handoff calls of MSs moving from a small cell to its associated sector \\
\hline
\end{tabular}


denote the total number of channels, i.e., time slots, frequency carriers, or timeand-frequency blocks, in the system. $N_{s r}\left(N_{s c}\right)$ is the total number of available channels in the same frequency band for a sector (small cell) region. There are two kinds of new calls (NCs), initiating in a sector region or a small cell coverage, designated by $N C_{s r}$ and $N C_{s o}$ respectively. Three kinds of handoff calls (HCs) are generated. One is from those MSs moving from a sector region to its embedded small cell, denoted as $H C_{s r_{-} s c}$. The second one is from those MSs moving in the reverse direction, denoted as $H C_{s c_{-} s r}$. The third one is from those MSs moving between two sectors, denoted as $H C_{s r_{-} s r}$.

$$
A r=\frac{\pi r^{2}}{\pi R^{2} / n}=\frac{n r^{2}}{R^{2}}
$$

\subsection{Handoff Rate}

As elaborated in Section 2.1, there are three kinds of handoff calls (designated by $H C_{s c_{-} s n} H C_{s r_{-} s o}$ and $\left.H C_{s r_{-} s r}\right)$ in the SCNSC. Referring to the works [28] [29], Equation (2) expresses the probability of an MS moving from a small cell area to its associated sector, and vice versa.

$$
\frac{E[V] \times L}{\pi \times A}
$$

where $L$ is the length of the boundary between a small cell and a sector, $E[V]$ is the average moving speed of an MS, and $A$ is the coverage area of a small cell or sector. As illustrated in Figure 4, three kinds of handoff rate are derived as follows.

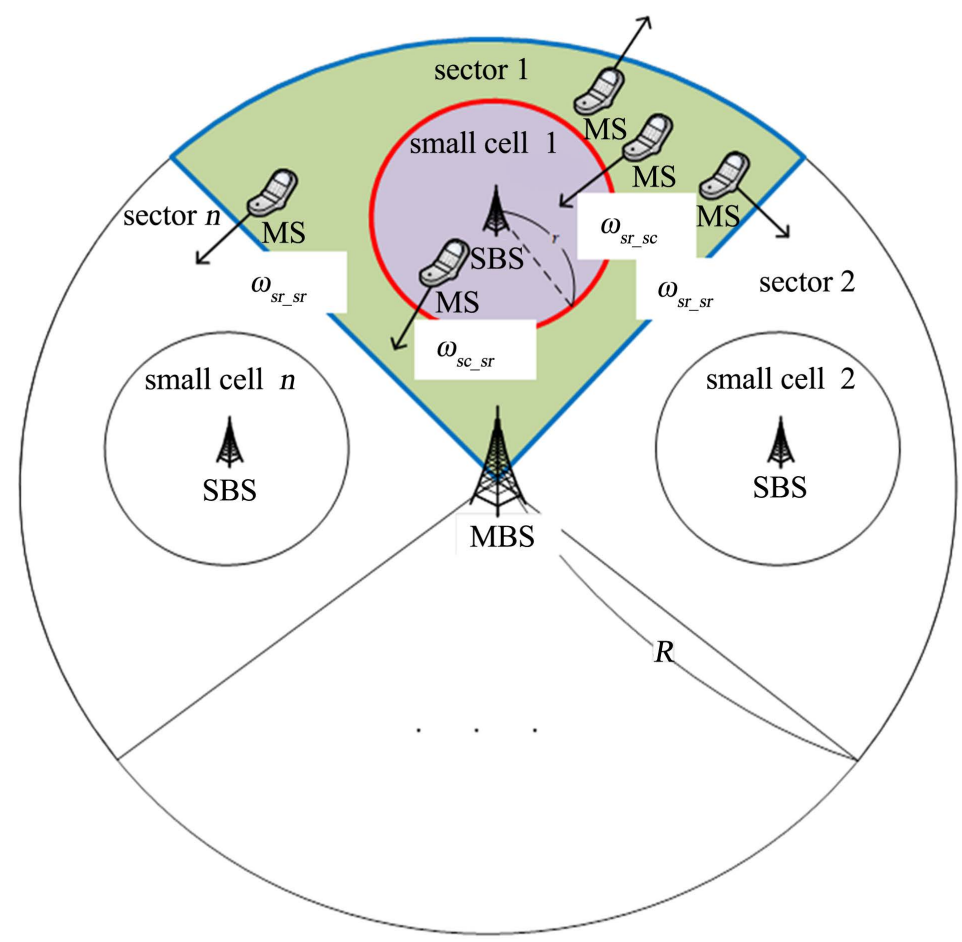

Figure 4. Three kinds of handoff in the SCNSC. 
1) Let $\omega_{s c_{-} s r}$ denote the handoff rate of $H C_{s c_{-} s r} . L$ and $A$ in Equation (2) are equal to $2 \pi r$ and $\pi r^{2}$, respectively. Then,

$$
\omega_{s c_{-} s r}=\frac{E[V] \times(2 \pi r)}{\pi \times\left(\pi r^{2}\right)}=\frac{2 \times E[V]}{\pi \times r}
$$

2) Let $\omega_{s r_{-} s c}$ denote the handoff rate of $H C_{s r_{-} s c}$. Similarly, $L$ and $A$ in Equation (2) are equal to $2 \pi r$ and $\pi R^{2} / n-\pi r^{2}$, respectively. Then,

$$
\omega_{s r_{-} s c}=\frac{E[V] \times(2 \pi r)}{\pi \times\left(\pi R^{2} / n-\pi r^{2}\right)}=\frac{2 \times E[V] \times r \times n}{\pi \times\left(R^{2}-n \times r^{2}\right)}
$$

3) Let $\omega_{s L_{-} s r}$ denote the handoff rate of $H C_{s r_{-} s r} A$ in Equation (2) is the same as that in Equation (4). $L$ represents the boundary of a sector, which is equal to $2 R+2 \pi R / n$. However, $H C_{s r_{-} s r}$ is defined as a handoff call when an MS moves from a sector to two adjacent sectors without including an MS moving out of the macro cell. Thus, $L$ in Equation (2) is modified as expressed in Equation (5). Replacing $A$ and $L$ in Equation (2) with the formulas for the kind of handoff, we obtain Equation (6).

$$
\begin{gathered}
L=(2 R+2 \pi R / n) \times(R /(2 R+2 \pi R / n)) \\
\omega_{s r_{-} s r}=\frac{E[V] \times(2 R+2 \pi R / n)}{\pi \times\left(\pi R^{2} / n-\pi r^{2}\right)} \times \frac{R}{2 R+2 \pi R / n}=\frac{E[V] \times R \times n}{\pi^{2} \times\left(R^{2}-n \times r^{2}\right)}
\end{gathered}
$$

\section{Analytical Model of the SCNSC}

In order to analyze the system performance of the proposed SCNSC, we build a mathematical model using Markov chain [30]. If $n$ is even, each state of the transition matrix is defined as a four-dimensional vector, denoted as $[i, j, x, y]$, in which $i(j)$ is the number of calls in the odd (even)-indexed sector and $x(y)$ is the number of calls in the odd (even)-indexed small cell. On the other hand, if $n$ is odd, each state of the transition matrix is defined as a six-dimensional vector, denoted as $[i, j, k, x, y, z]$, in which $i(j$ or $k)$ is the number of calls in the sector indexed by a multiple of three plus one (two or zero) and $x$ ( $y$ or $z$ ) is the number of calls in the small cell indexed by a multiple of three plus one (two or zero). Firstly, we calculate the transition rates, which contain the arrival and departure rates between any two of the states. Then, each state probability is obtained and the performance measures, including the NC blocking and HC dropping probabilities, the average waiting time, and the normalized throughput, are derived in terms of the obtained state probabilities.

\subsection{Assumptions}

1) MSs are homogeneously distributed in a macro cell.

2) Each new/handoff call occupies one channel.

3) The average moving speed of MSs in either of a sector or a small cell is the same.

4) The system bandwidth is channelized with a maximum number, denoted as 
$N$.

Under the above assumptions, the number of available channels in the same frequency band for a small cell, denoted as $N_{s o}$ can be expressed by Equation (7). Moreover, the number of available channels in the same frequency band for a sector region excluding its embedded cell coverage, denoted as $N_{s D}$ is expressed as Equation (8).

$$
\begin{gathered}
N_{s c}=\frac{N}{n_{f}} \times A r \\
N_{s r}=\frac{N}{n_{f}} \times(1-A r)
\end{gathered}
$$

\subsection{Analytical Model}

\subsubsection{Transition Rates}

The transition rates in the Markov model include arrival rate, denoted by $\lambda$, and departure rate, denoted by $\mu$. The arrival rate is composes of those from NCs and $\mathrm{HCs}$, while the departure rate is generated from call handoff or termination. The arrival rates associated with NCs, consisting of $N C_{s c}$ and $N C_{s n}$ are denoted as $\lambda_{s c}$ and $\lambda_{s r}$, respectively. The arrival rates from HCs, consisting of $H C_{s r_{-} s r} H C_{s I_{-} s o}$ and $H C_{s c_{-} s n}$ are denoted by $\lambda_{s r_{-} s n} \lambda_{s r_{-} s o}$ and $\lambda_{s c_{-} s n}$ respectively. Similarly, the departure rates caused by call handoff, consisting of $H C_{s r_{-} s l} H C_{s r_{-} s o}$ and $H C_{s C_{-} s l}$ are denoted as $\mu_{s L_{-} s s} \mu_{s L_{-} s o}$ and $\mu_{\left.s c_{-} s\right)}$ respectively. The departure rates caused by call termination include $\mu_{s c}$ and $\mu_{s s}$ which stand for the calls terminating in a small cell and a sector region, respectively. $\mu_{s c}$ contains two parts: one, denoted as $\mu_{s c_{-} t}$ is for NCc originally initiating in a small cell and the other, denoted as $\mu_{s \tau_{-} s c_{-} b}$ is for $H C_{s r_{s} s c}$ terminating in a small cell. $\mu_{s r}$ contains three parts: one, denoted as $\mu_{s r_{-} b}$ is for NCs originally initiating in a sector and the other two, denoted as $\mu_{s C_{-} r_{-} t}$ and $\mu_{s \Gamma_{-} s_{-} t}$, respectively for $H C_{s C_{-} s r}$ and $H C_{s r_{-} s r}$ terminating in a sector. Table 2 summarizes the abovementioned transition rates in the Markov model.

Therefore, $\lambda_{s c}$ and $\lambda_{s r}$ can be expressed by Equations (9) and (10), respectively. In the balanced condition of the Markov model, the arrival rate and departure rate caused by call handoff is the same. That's, $\lambda_{s r_{-} s t} \lambda_{s r_{-} s o}$ and $\lambda_{s c_{-} s r}$ are equal to $\mu_{s r_{-} s s} \mu_{s I_{-} s o}$ and $\mu_{s c_{-} s r}$ respectively. We can obtain the handoff-call arrival (departure) rate for each state by multiplying the varied element in the state by the associated handoff rate, as derived in Section 2.2. As to the departure rate caused by call termination in a small cell coverage $\left(\mu_{s c}\right)$ is the sum of $\mu_{s c_{-} t}$ and $\mu_{s r_{-} s c_{-} b}$ which are expressed by Equations (11) and (12), respectively. Similarly, the departure rate caused by call termination in a sector region $\left(\mu_{s r}\right)$ is the sum of $\mu_{s L_{-} t}$ $\mu_{s c_{\_} s r_{-} b}$ and $\mu_{s r_{-} s r_{-} b}$ which are expressed in Equations (13) to (15).

$$
\begin{gathered}
\lambda_{s c}=\lambda \times A r \\
\lambda_{s r}=\lambda \times(1-A r) \\
\mu_{s c_{-} t}=\mu \times A r \times \frac{\lambda_{s c}}{\lambda_{s c}+\lambda_{s r_{-} s c}}
\end{gathered}
$$


Table 2. Arrival rates and departure rates in the Markov model.

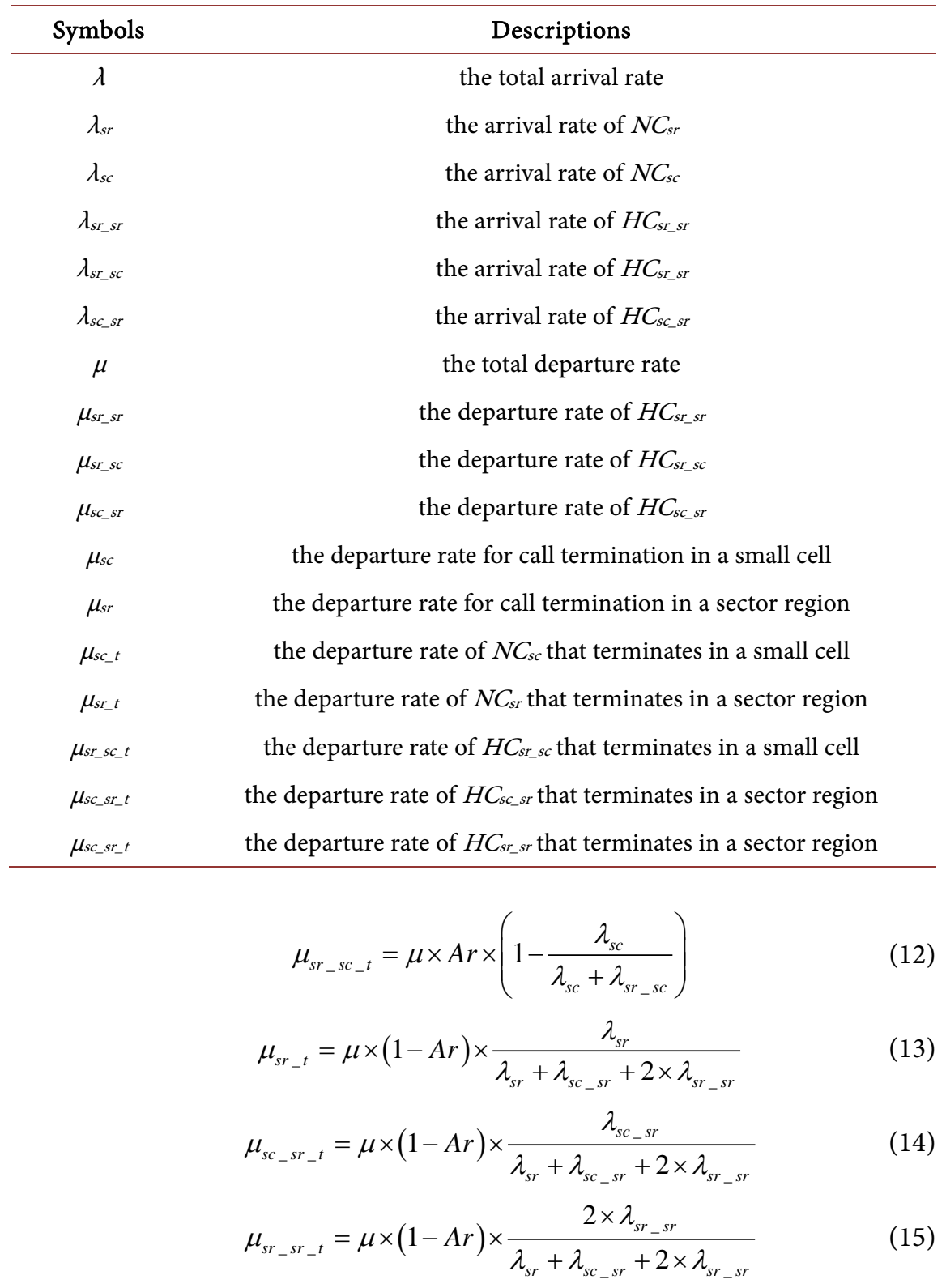

\subsubsection{Markov States}

All of the states in the Markov model are composed of the following five portions:

1) The number of calls in either a small cell or a sector does not exceed the upper limit. That's, each sector-related (small-cell-related) element of a state varies from 0 to $N_{s r}\left(N_{s c}\right)$ and there are $n_{f}$ frequency bands. Therefore, the total number of states in this portion, denoted as $N_{1}$, is calculated as below.

$$
N_{1}=\left(N_{s r}+1\right)^{n_{f}} \times\left(N_{s c}+1\right)^{n_{f}}
$$

2) NC in a small cell being blocked: when the number of calls in a small cell reaches the maximum value, i.e., $N_{s o}$ an NC will be blocked. The total number of states in the portion, denoted as $N_{2}$, is calculated as Equation (17). 


$$
N_{2}=\left(N_{s r}+1\right)^{n_{f}} \times\left(N_{s c}+1\right)^{n_{f}-1} \times n_{f}
$$

3) NC in a sector being blocked: when the number of calls in a sector reaches the maximum value, i.e., $N_{s r}$ an NC will be blocked. The total number of states in the portion, denoted as $N_{3}$, is calculated as Equation (18).

$$
N_{3}=\left(N_{s r}+1\right)^{n_{f}-1} \times\left(N_{s c}+1\right)^{n_{f}} \times n_{f}
$$

4) HC moving into a small cell being dropped: when the number of calls in a small cell reaches the maximum value $\left(N_{s c}\right)$, an $\mathrm{HC}$ will be dropped. The total number of states in the portion, denoted as $N_{4}$, is calculated as Equation (19).

$$
N_{4}=N_{s r} \times\left(N_{s r}+1\right)^{n_{f}-1} \times\left(N_{s c}+1\right)^{n_{f}-1} \times n_{f}
$$

5) HC moving into a sector being dropped: there are two situations in the portion of states. That's, an HC is moving from a small cell to a sector and an $\mathrm{HC}$ is moving from a sector to two adjacent sectors. The total number of states in the portion, denoted as $N_{5}$, is calculated as Equation (20) depending on the number of sectors (denoted by $n$ ), is even or odd.

$$
N_{5}=\left\{\begin{array}{l}
\left(N_{s r}+1\right)^{n_{f}-1} \times\left(N_{s c}+1\right)^{n_{f}-1} \times N_{s c} \times n_{f}+N_{s r} \times\left(N_{s c}+1\right)^{n_{f}} \times n_{f} \\
-\left(N_{s c}+1\right) \times N_{s c} \times N_{s r} \times n_{f}, \text { if } n \text { is even } \\
\left(N_{s r}+1\right)^{n_{f}-1} \times\left(N_{s c}+1\right)^{n_{f}-1} \times N_{s c} \times n_{f} \\
+\left(N_{s r} \times 2\left(N_{s r}+1\right)-N_{s r}^{2}\right) \times\left(N_{s c}+1\right)^{n_{f}} \times n_{f} \\
-\left(N_{s c}+1\right)^{n_{f}-1} \times\left(\left(N_{s r}+1\right)^{n_{f}-1}-1\right) \times n_{f}, \text { if } n \text { is odd }
\end{array}\right.
$$

By summing Equations (16) to (20), we can obtain the total number of the states, $N$, as expressed in Equation (21). The size of the transition matrix for the Markov model is $N_{t} \times N_{t}$.

$$
N_{t}=N_{1}+N_{2}+N_{3}+N_{4}+N_{5}
$$

\subsection{Performance Measures}

\subsubsection{NC Blocking and HC Dropping Probabilities}

An NC may get rejected in a small cell if one of the small-cell-related elements in the current state is $N_{s c}$ and one more NC makes the element become $N_{s c}+1$. Thus, the NC blocking probability in a small cell, denoted as $P b_{s o}$ is expressed as Equation (22). Similarly, an NC may get rejected in a sector if one of the sector-related elements in the current state is $N_{s r}$. Thus, the NC blocking probability in a sector, denoted as $P b_{s n}$ is expressed by Equation (23).

$$
\begin{aligned}
& P b_{s c}=\left\{\begin{array}{l}
\sum_{i=0}^{N_{s r}} \sum_{j=0}^{N_{s r}} \sum_{y=0}^{N_{s c}} P\left(i, j, N_{s c}+1, y\right) \times n_{f}, \text { if } n \text { is even } \\
\sum_{i=0}^{N_{s r}} \sum_{j=0}^{N_{s r}} \sum_{k=0}^{N_{s r}} \sum_{y=0}^{N_{S c}} \sum_{z=0}^{N_{s c}} P\left(i, j, k, N_{s c}+1, y, z\right) \times n_{f}, \text { if } n \text { is odd }
\end{array}\right. \\
& P b_{s r}=\left\{\begin{array}{l}
\sum_{j=0}^{N_{s r}} \sum_{x=0}^{N_{s c}} \sum_{y=0}^{N_{s c}} P\left(N_{s r}+1, j, x, y\right) \times n_{f}, \text { if } n \text { is even } \\
\sum_{j=0}^{N_{s r}} \sum_{k=0}^{N_{s r}} \sum_{x=0}^{N_{S c}} \sum_{y=0}^{N_{s c}} \sum_{z=0}^{N_{s c}} P\left(N_{s r}+1, j, k, x, y, z\right) \times n_{f}, \text { if } n \text { is odd }
\end{array}\right.
\end{aligned}
$$


On the other hand, an HC moving from a sector to a small cell gets no services when one of the small-cell-related elements in the current state reaches $N_{s c}$. one more HC makes the element become $\left(N_{s c}+1\right)^{\prime}$. We use the symbol prime to indicate the case when the arriving call belongs to HCs. Therefore, the HC dropping probability for MSs moving from a sector to a small cell, denoted by $P d_{s r_{-} s o}$ is calculated as Equation (24).

$$
P d_{s r_{-} s c}=\left\{\begin{array}{l}
\sum_{i=0}^{N_{s r}} \sum_{j=0}^{N_{s r}} \sum_{y=0}^{N_{s c}} P\left(i, j,\left(N_{s c}+1\right)^{\prime}, y\right) \times n_{f}, \text { if } n \text { is even } \\
\sum_{i=0}^{N_{s r}} \sum_{j=0}^{N_{s r}} \sum_{k=0}^{N_{s r}} \sum_{y=0}^{N_{s c}} \sum_{z=0}^{N_{s c}} P\left(i, j, k,\left(N_{s c}+1\right)^{\prime}, y, z\right) \times n_{f}, \text { if } n \text { is odd }
\end{array}\right.
$$

In addition, there are two cases of an HC getting no services in a sector. One is for an HC moving into a sector from its embedded cell, and the other is for an HC moving from either of two adjacent sectors. Let $P d_{s c_{-} s r}$ and $P d_{s r_{-} s r}$ be the HC dropping probabilities in the two cases. Equations (25) and (26) express the two cases, respectively.

$$
P d_{s c_{-} s r}=\left\{\begin{array}{l}
\sum_{x=0}^{N_{s c}} \sum_{y=0}^{N_{s c}-1}\left[\sum_{j=0}^{N_{s r}-1} P\left(\left(N_{s r}+1\right)^{\prime}, j, x, y\right) \times \frac{\lambda_{s c_{-} s r}}{2 \lambda_{s r_{-} s r}+\lambda_{s c_{-} s r}}\right. \\
\left.+P\left(\left(N_{s r}+1\right)^{\prime}, N_{s r}, x, y\right)\right] \times n_{f}, \text { if } n \text { is even } \\
\sum_{x=0}^{N_{s c}} \sum_{y=0}^{N_{s c}} \sum_{z=0}^{N_{s c}-1}\left[\sum_{j=0}^{N_{s r}-1} \sum_{k=0}^{N_{s r}-1} P\left(\left(N_{s r}+1\right)^{\prime}, j, k, x, y, z\right) \times \frac{\lambda_{s c_{-} s r}}{2 \lambda_{s r_{-} s r}+\lambda_{s c_{-} s r}}\right. \\
+\sum_{k=0}^{N_{s r}-1} P\left(\left(N_{s r}+1\right)^{\prime}, N_{s r}, k, x, y, z\right) \times \frac{2 \lambda_{s c_{-} s r_{1}}}{\lambda_{s r_{-} s r}+\lambda_{s c_{-} s r}} \\
\left.+P\left(\left(N_{s r}+1\right)^{\prime}, N_{s r}, N_{s r}, x, y, z\right)\right] \times n_{f}, \text { if } n \text { is odd }
\end{array}\right.
$$

where $\frac{\lambda_{s c_{-} s r}}{2 \times \lambda_{s r_{-} s r}+\lambda_{s c_{-} s r}}$ stands for the proportion of HCs moving into a sector from its embedded cell because an $\mathrm{HC}$ in a sector may arrive from an embedded cell or two adjacent sectors if $n$ is even.

As to the metric $P d_{s r_{-} s r}$ it is straightforward equal to the HC dropping probability in a sector excluding $P d_{s c_{-} s}$.

$$
P d_{s r_{-} s r}=\left\{\begin{array}{l}
\sum_{j=0}^{N_{s r}} \sum_{x=0}^{N_{s c}} \sum_{y=0}^{N_{s c}} P\left(\left(N_{s r}+1\right)^{\prime}, j, x, y\right) \times n_{f}-P d_{s c_{-} s r}, \text { if } n \text { is even } \\
\sum_{j=0}^{N_{s r}} \sum_{k=0}^{N_{s r}} \sum_{x=0}^{N_{s c}} \sum_{y=0}^{N_{s c}} \sum_{z=0}^{N_{s c}} P\left(\left(N_{s r}+1\right)^{\prime}, j, k, x, y, z\right) \times n_{f}-P d_{s c_{-} s r}, \text { if } n \text { is odd }
\end{array}\right.
$$

\subsubsection{Average Waiting Time}

Before deriving the average waiting time for an MS, it is required to obtain the average queue length (AQL) in a sector or a small cell. The AQL of NCs in a sector, denoted as $Q n_{s r}$, is the product of $N_{s r}+1$ and the summation of the states 
an NC is blocked in the sector, as expressed in Equation (27). Similarly, we can obtain the AQL of HCs in a sector, denoted as $Q h_{s p}$ by multiplying $N_{s r}+1$ by the summation of the states an $\mathrm{HC}$ is dropped in the sector, as expressed in Equation (28). Similar to Equations (27) and (28), the AQL of NCs and HCs in a small cell, can be expressed by Equations (29) and (30), respectively.

$$
\begin{aligned}
& Q n_{s r}=\left\{\begin{array}{l}
\sum_{j=0}^{N_{s r}} \sum_{x=0}^{N_{s c}} \sum_{y=0}^{N_{s c}}\left(N_{s r}+1\right) \times P\left(N_{s r}+1, j, x, y\right), \text { if } n \text { is even } \\
\sum_{j=0}^{N_{s r}} \sum_{k=0}^{N_{s r}} \sum_{x=0}^{N_{s c}} \sum_{y=0}^{N_{s c}} \sum_{z=0}^{N_{s c}}\left(N_{s r}+1\right) \times P\left(N_{s r}+1, j, k, x, y, z\right), \text { if } n \text { is odd }
\end{array}\right. \\
& Q h_{s r}=\left\{\begin{array}{l}
\sum_{j=0}^{N_{s r}} \sum_{x=0}^{N_{s c}} \sum_{y=0}^{N_{s c}}\left(N_{s r}+1\right) \times P\left(\left(N_{s r}+1\right)^{\prime}, j, x, y\right), \text { if } n \text { is even } \\
\sum_{j=0}^{N_{s r}} \sum_{k=0}^{N_{s r}} \sum_{x=0}^{N_{s c}} \sum_{y=0}^{N_{s c}} \sum_{z=0}^{N_{s c}}\left(N_{s r}+1\right) \times P\left(\left(N_{s r}+1\right)^{\prime}, j, k, x, y, z\right), \text { if } n \text { is odd }
\end{array}\right. \\
& Q n_{s c}=\left\{\begin{array}{l}
\sum_{i=0}^{N_{s r}} \sum_{j=0}^{N_{s r}} \sum_{y=0}^{N_{s c}}\left(N_{s c}+1\right) \times P\left(i, j, N_{s c}+1, y\right), \text { if } n \text { is even } \\
\sum_{i=0}^{N_{s r}} \sum_{j=0}^{N_{s r}} \sum_{k=0}^{N_{s r}} \sum_{y=0}^{N_{s c}} \sum_{z=0}^{N_{s c}}\left(N_{s c}+1\right) \times P\left(i, j, k, N_{s c}+1, y, z\right), \text { if } n \text { is odd }
\end{array}\right. \\
& Q h_{s c}=\left\{\begin{array}{l}
\sum_{i=0}^{N_{s r}} \sum_{j=0}^{N_{s r}} \sum_{y=0}^{N_{s c}}\left(N_{s c}+1\right) \times P\left(i, j,\left(N_{s c}+1\right)^{\prime}, y\right), \text { if } n \text { is even } \\
\sum_{i=0}^{N_{s r}} \sum_{j=0}^{N_{s r}} \sum_{k=0}^{N_{s r}} \sum_{y=0}^{N_{s c}} \sum_{z=0}^{N_{s c}}\left(N_{s c}+1\right) \times P\left(i, j, k,\left(N_{s c}+1\right)^{\prime}, y, z\right), \text { if } n \text { is odd }
\end{array}\right.
\end{aligned}
$$

Referring to Equation (27), the state probability $P\left(N_{s r}+1, j, x, y\right)$ in $Q n_{s r}$ contains two sets: $N_{s r}$ ongoing calls that are composed of $\lambda_{s r} \lambda_{s C_{C} s r}$ and $\lambda_{s L_{-} s r}$ and one NC initiating in a sector that may get blocked. As a result, the average waiting time (AWT) of NCs in a sector, denoted as $W n_{s l}$ can be expressed by Equation (31). On the other hand, the average waiting time (AWT) of HCs in a sector, denoted as $W h_{s r}$ is derived as Equation (32) by referring to Equation (28). The state probability $P\left(N_{s r}+1, j, x, y\right)$ in $Q h_{s r}$ contains two sets: $N_{s r}$ ongoing calls that are composed of $\lambda_{s r} \lambda_{s c_{-} s r}$ and $\lambda_{s r_{-} s r}$ and one HC generating from $\lambda_{s c_{-} s r}$ and $\lambda_{s r_{s} r}$ that may get dropped. Similarly, we can derive the AWT of NCs and HCs in a small cell, respectively denoted as $W n_{s c}$ and $W h_{s o}$ by referring to Equations (29) and (30).

$$
W n_{s r}=\left\{\begin{array}{l}
\sum_{j=0}^{N_{s r}} \sum_{x=0}^{N_{s c}} \sum_{y=0}^{N_{s c}} \frac{P\left(N_{s r}+1, j, x, y\right)}{\lambda_{s r}} \\
+\sum_{j=0}^{N_{s r}} \sum_{x=0}^{N_{s c}} \sum_{y=0}^{N_{s c}} \frac{N_{s r} \times P\left(N_{s r}+1, j, x, y\right)}{\lambda_{s r}+\lambda_{s c_{-} s r}+2 \lambda_{s r_{-} s r}}, \text { if } n \text { is even } \\
\sum_{j=0}^{N_{s r}} \sum_{k=0}^{N_{s r}} \sum_{x=0}^{N_{s c}} \sum_{y=0}^{N_{s c}} \sum_{z=0}^{N_{s c}} \frac{P\left(N_{s r}+1, j, k, x, y, z\right)}{\lambda_{s r}} \\
+\sum_{j=0}^{N_{s r}} \sum_{k=0}^{N_{s r}} \sum_{x=0}^{N_{s c}} \sum_{y=0}^{N_{s c}} \sum_{z=0}^{N_{s c}} \frac{N_{s r} \times P\left(N_{s r}+1, j, k, x, y, z\right)}{\lambda_{s r}+\lambda_{s c_{-} s r}+2 \lambda_{s r_{-} s r}}, \text { if } n \text { is odd }
\end{array}\right.
$$




$$
\begin{aligned}
& \left(\sum_{j=0}^{N_{s r}} \sum_{x=0}^{N_{s c}} \sum_{y=0}^{N_{s c}} \frac{P\left(\left(N_{s r}+1\right)^{\prime}, j, x, y\right)}{\lambda_{s c_{-} s r}+2 \lambda_{s r_{-} s r}}\right. \\
& W h_{s r}=\left\{\begin{array}{l}
+\sum_{j=0}^{N_{s r}} \sum_{x=0}^{N_{s c}} \sum_{y=0}^{N_{s c}} \frac{N_{s r} \times P\left(\left(N_{s r}+1\right)^{\prime}, j, x, y\right)}{\lambda_{s r}+\lambda_{s c_{-} s r}+2 \lambda_{s r_{-} s r}}, \text { if } n \text { is even } \\
N_{s r} N_{s r} N_{s c} N_{s c} N_{s c} P\left(\left(N_{s r}+1\right)^{\prime}, j, k, x, y, z\right)
\end{array}\right. \\
& \sum_{j=0}^{N_{s r}} \sum_{k=0}^{N_{s r}} \sum_{x=0}^{N_{s c}} \sum_{y=0}^{N_{s c}} \sum_{z=0}^{N_{s c}} \frac{P\left(\left(N_{s r}+1\right)^{\prime}, j, k, x, y, z\right)}{\lambda_{s c_{-} s r}+2 \lambda_{s r_{-} s r}} \\
& +\sum_{j=0}^{N_{s r}} \sum_{k=0}^{N_{s r}} \sum_{x=0}^{N_{s c}} \sum_{y=0}^{N_{s c}} \sum_{z=0}^{N_{s c}} \frac{N_{s r} \times P\left(\left(N_{s r}+1\right)^{\prime}, j, k, x, y, z\right)}{\lambda_{s r}+\lambda_{s c_{-} s r}+2 \lambda_{s r_{-} s r}} \text {, if } n \text { is odd } \\
& \left(\sum_{i=0}^{N_{s r}} \sum_{j=0}^{N_{s r}} \sum_{y=0}^{N_{s c}} \frac{P\left(i, j, N_{s c}+1, y\right)}{\lambda_{s c}}\right. \\
& W n_{s c}=\left\{\begin{array}{l}
+\sum_{i=0}^{N_{s r}} \sum_{j=0}^{N_{s r}} \sum_{y=0}^{N_{s c}} \frac{N_{s c} \times P\left(i, j, N_{s c}+1, y\right)}{\lambda_{s c}+\lambda_{s r_{-} s c}}, \text { if } n \text { is even } \\
N_{s r} N_{s r} N_{s r} N_{s c} N_{s c} P\left(i, j, k, N_{s c}+1, y, z\right)
\end{array}\right. \\
& \sum_{i=0}^{N_{s r}} \sum_{j=0}^{N_{s r}} \sum_{k=0}^{N_{s r}} \sum_{y=0}^{N_{s c}} \sum_{z=0}^{N_{s c}} \frac{P\left(i, j, k, N_{s c}+1, y, z\right)}{\lambda_{s c}} \\
& +\sum_{i=0}^{N_{s r}} \sum_{j=0}^{N_{s r}} \sum_{k=0}^{N_{s r}} \sum_{y=0}^{N_{s c}} \sum_{z=0}^{N_{s c}} \frac{N_{s c} \times P\left(i, j, k, N_{s c}+1, y, z\right)}{\lambda_{s c}+\lambda_{s r_{-} s c}} \text {, if } n \text { is odd } \\
& W h_{s c}=\left\{\begin{array}{l}
\sum_{i=0}^{N_{s r}} \sum_{j=0}^{N_{s r}} \sum_{y=0}^{N_{s c}} \frac{P\left(i, j,\left(N_{s c}+1\right)^{\prime}, y\right)}{\lambda_{s r_{-} s c}} \\
+\sum_{i=0}^{N_{s r}} \sum_{j=0}^{N_{s r}} \sum_{y=0}^{N_{s c}} \frac{N_{s c} \times P\left(i, j,\left(N_{s c}+1\right)^{\prime}, y\right)}{\lambda_{s c}+\lambda_{s r_{-} s c}}, \text { if } n \text { is even } \\
\sum_{i=0}^{N_{s r}} \sum_{j=0}^{N_{s r}} \sum_{k=0}^{N_{s r}} \sum_{y=0}^{N_{s c}} \sum_{z=0}^{N_{s c}} \frac{P\left(i, j, k,\left(N_{s c}+1\right)^{\prime}, y, z\right)}{\lambda_{s r_{-} s c}} \\
+\sum_{i=0}^{N_{s r}} \sum_{j=0}^{N_{s r}} \sum_{k=0}^{N_{s r}} \sum_{y=0}^{N_{s c}} \sum_{z=0}^{N_{s c}} \frac{N_{s c} \times P\left(i, j, k,\left(N_{s c}+1\right)^{\prime}, y, z\right)}{\lambda_{s c}+\lambda_{s r_{-} s c}}, \text { if } n \text { is odd }
\end{array}\right.
\end{aligned}
$$

\subsubsection{Normalized Throughput}

We define the normalized throughput (NT) as the individual channel utilization for a sector, a small cell, or a macro cell. Let $\Gamma_{s r}$ be the NT for a sector. As expressed in Equation (35), $\Gamma_{s r}$ consists of three conditions: one is for the states the channels are available for both in a sector and a small cell (i.e., the first term of Equation (35)); one is for the states the channels are fully occupied in a sector and an NC or HC gets no services in a sector (i.e., the second and third terms of Equation (35)); the other one is for the states the channels are fully occupied in a small cell and an NC or HC gets no services in a small cell (i.e., the fourth and fifth terms of Equation (35)). Similar to the derivation of $\Gamma_{s n}$ we obtain the NT for a small cell, denoted as $\Gamma_{s o}$ as expressed in Equation (36). Finally, let $\Gamma_{m c}$ be the NT for the macro cell. Similarly, we can obtain $\Gamma_{m o}$ as expressed by Equation (37). 


$$
\begin{aligned}
& \Gamma_{s r}=\left\{\begin{array}{l}
\sum_{i=0}^{N_{s r}} \sum_{j=0}^{N_{s r}} \sum_{x=0}^{N_{s c}} \sum_{y=0}^{N_{s c}} \frac{i+j}{N_{s r} \times n_{f}} \times P(i, j, x, y) \\
+\left[\sum_{j=0}^{N_{s r}} \sum_{x=0}^{N_{s c}} \sum_{y=0}^{N_{s c}} \frac{N_{s r}+j}{N_{s r} \times n_{f}} \times P\left(N_{s r}+1, j, x, y\right)\right. \\
\left.+\sum_{j=0}^{N_{s r}} \sum_{x=0}^{N_{s c}} \sum_{y=0}^{N_{s c}} \frac{N_{s r}+j}{N_{s r} \times n_{f}} \times P\left(\left(N_{s r}+1\right)^{\prime}, j, x, y\right)\right] \times n_{f} \\
+\left[\sum_{i=0}^{N_{s r}} \sum_{j=0}^{N_{s r}} \sum_{y=0}^{N_{s c}} \frac{i+j}{N_{s r} \times n_{f}} \times P\left(i, j, N_{s c}+1, y\right)\right. \\
\left.+\sum_{i=0}^{N_{s r}} \sum_{j=0}^{N_{s r}} \sum_{y=0}^{N_{s c}} \frac{i+j}{N_{s r} \times n_{f}} \times P\left(i, j,\left(N_{s c}+1\right)^{\prime}, y\right)\right] \times n_{f}, \text { if } n \text { is even } \\
\sum_{i=0}^{N_{s r}} \sum_{j=0}^{N_{s r}} \sum_{k=0}^{N_{s r}} \sum_{x=0}^{N_{s c}} \sum_{y=0}^{N_{s c}} \sum_{z=0}^{N_{s c}} \frac{i+j+k}{N_{s r} \times n_{f}} \times P(i, j, k, x, y, z) \\
+\left[\sum_{j=0}^{N_{s r}} \sum_{k=0}^{N_{s r}} \sum_{x=0}^{N_{s c}} \sum_{y=0}^{N_{s c}} \sum_{z=0}^{N_{s c}} \frac{N_{s r}+j+k}{N_{s r} \times n_{f}} \times P\left(N_{s r}+1, j, k, x, y, z\right)\right. \\
\left.+\sum_{j=0}^{N_{s r}} \sum_{k=0}^{N_{s r}} \sum_{x=0}^{N_{s c}} \sum_{y=0}^{N_{s c}} \sum_{z=0}^{N_{s c}} \frac{N_{s r}+j+k}{N_{s r} \times n_{f}} \times P\left(\left(N_{s r}+1\right)^{\prime}, j, k, x, y, z\right)\right] \times n_{f} \\
+\left[\sum_{j=0}^{N_{s r}} \sum_{k=0}^{N_{s r}} \sum_{x=0}^{N_{s c}} \sum_{y=0}^{N_{s c}} \sum_{z=0}^{N_{s c}} \frac{i+j+k}{N_{s r} \times n_{f}} \times P\left(i, j, k, N_{s c}+1, y, z\right)\right. \\
\left.+\sum_{j=0}^{N_{s r}} \sum_{k=0}^{N_{s r}} \sum_{x=0}^{N_{s c}} \sum_{y=0}^{N_{s c}} \sum_{z=0}^{N_{s c}} \frac{i+j+k}{N_{s r} \times n_{f}} \times P\left(i, j, k,\left(N_{s c}+1\right)^{\prime}, y, z\right)\right] \times n_{f}, \text { if } n \text { is odd }
\end{array}\right.
\end{aligned}
$$

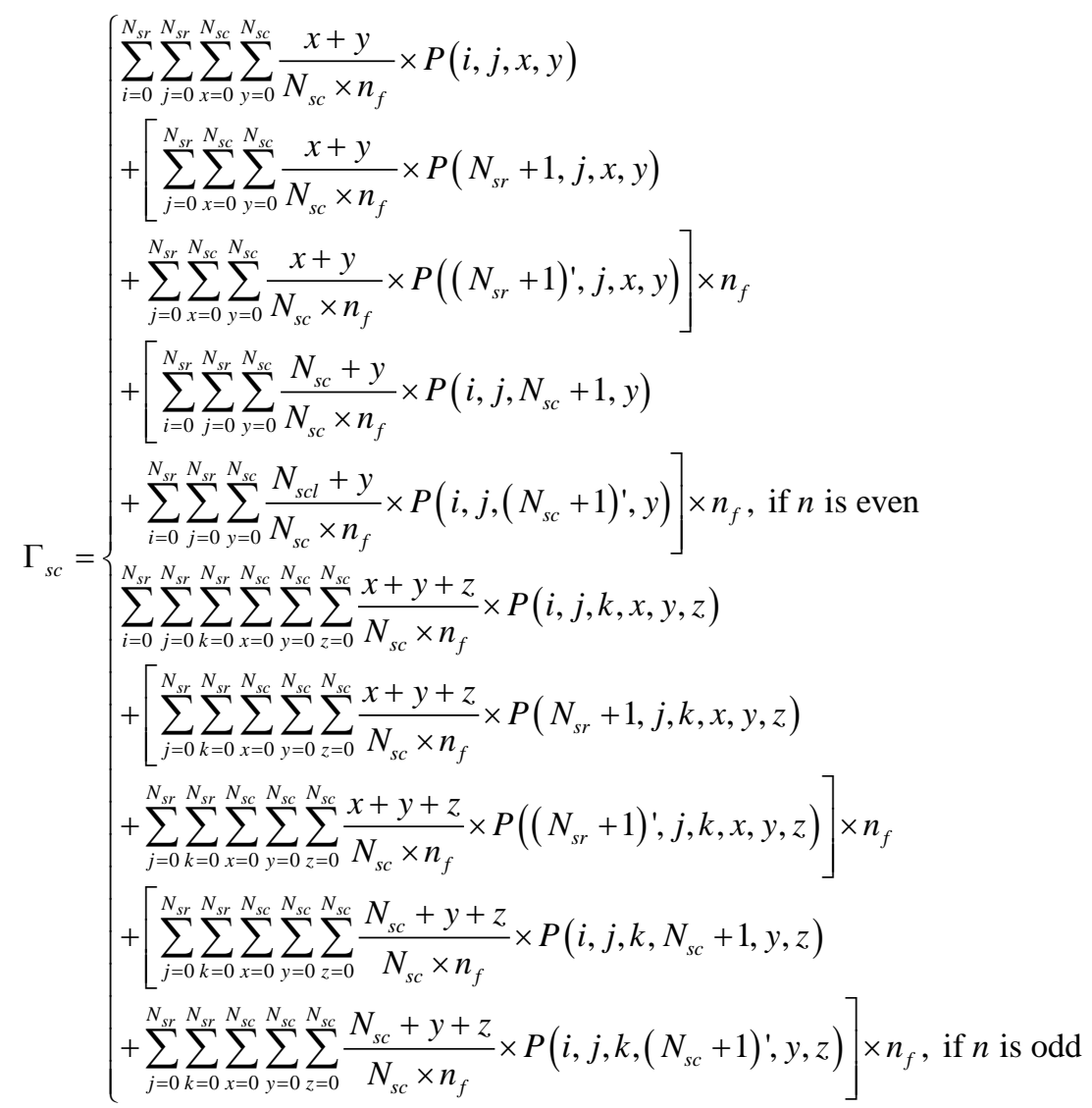




$$
\begin{aligned}
& \left(\sum_{i=0}^{N_{S r}} \sum_{j=0}^{N_{S r}} \sum_{x=0}^{N_{S C}} \sum_{y=0}^{N_{S C}} \frac{i+j+x+y}{N} \times P(i, j, x, y)\right. \\
& +\left[\sum_{j=0}^{N_{s r}} \sum_{x=0}^{N_{s c}} \sum_{y=0}^{N_{s c}} \frac{N_{s r}+j+x+y}{N} \times P\left(N_{s r}+1, j, x, y\right)\right. \\
& \left.+\sum_{j=0}^{N_{s r}} \sum_{x=0}^{N_{s c}} \sum_{y=0}^{N_{s c}} \frac{N_{s r}+j+x+y}{N} \times P\left(\left(N_{s r}+1\right)^{\prime}, j, x, y\right)\right] \times n_{f} \\
& +\left[\sum_{i=0}^{N_{s r}} \sum_{j=0}^{N_{s r}} \sum_{y=0}^{N_{s c}} \frac{i+j+N_{s c}+y}{N} \times P\left(i, j, N_{s c}+1, y\right)\right. \\
& \left.+\sum_{i=0}^{N_{s r}} \sum_{j=0}^{N_{s r}} \sum_{y=0}^{N_{s c}} \frac{i+j+N_{s c}+y}{N} \times P\left(i, j,\left(N_{s c}+1\right)^{\prime}, y\right)\right] \times n_{f} \text {, if } n \text { is even } \\
& \sum_{i=0}^{N_{S r}} \sum_{j=0}^{N_{S r}} \sum_{k=0}^{N_{S r}} \sum_{x=0}^{N_{S C}} \sum_{y=0}^{N_{S C}} \sum_{z=0}^{N_{S C}} \frac{i+j+k+x+y+z}{N} \times P(i, j, k, x, y, z) \\
& +\left[\sum_{j=0}^{N_{s r}} \sum_{k=0}^{N_{s r}} \sum_{x=0}^{N_{s c}} \sum_{y=0}^{N_{s c}} \sum_{z=0}^{N_{s c}} \frac{N_{s r}+j+k+x+y+z}{N} \times P\left(N_{s r}+1, j, k, x, y, z\right)\right. \\
& \left.+\sum_{j=0}^{N_{s r}} \sum_{k=0}^{N_{s r}} \sum_{x=0}^{N_{S C}} \sum_{y=0}^{N_{S C}} \sum_{z=0}^{N_{S c}} \frac{N_{s r}+j+k+x+y+z}{N} \times P\left(\left(N_{s r}+1\right)^{\prime}, j, k, x, y, z\right)\right] \times n_{f} \\
& +\left[\sum_{j=0}^{N_{s r}} \sum_{k=0}^{N_{S r}} \sum_{x=0}^{N_{S C}} \sum_{y=0}^{N_{S C}} \sum_{z=0}^{N_{S C}} \frac{i+j+k+N_{s c}+y+z}{N} \times P\left(i, j, k, N_{s c}+1, y, z\right)\right. \\
& \left.+\sum_{j=0}^{N_{s r}} \sum_{k=0}^{N_{s r}} \sum_{x=0}^{N_{S C}} \sum_{y=0}^{N_{S C}} \sum_{z=0}^{N_{s C}} \frac{i+j+k+N_{s c}+y+z}{N} \times P\left(i, j, k,\left(N_{s c}+1\right)^{\prime}, y, z\right)\right] \times n_{f} \text {, if } n \text { is odd }
\end{aligned}
$$

\section{Analytical Results}

\subsection{Parameters and Values}

We evaluate the system performance of our proposed SCNSC via the analytical model built in Section 3. By setting the parameters as shown in Table 3, the transmission radius of the macro cell is fixed at 1000 meters and the transmission radius of a small cell is varied to change its coverage. The total number of channels in the system is given 60 and the number of sectors is set to be 3 or 6 . In order to observe the effect of the average moving speed $(E[V])$ of MSs on the

Table 3. Parameters and their values.

\begin{tabular}{cc}
\hline Parameters & Values \\
$R$ & 1000 meters \\
$r$ & $50,150,250$ meters \\
$N$ & 60 \\
$n$ & 3,6 \\
$E[V]$ & $20,40,60 \mathrm{~km} / \mathrm{hr}$ \\
$\lambda$ & $0.001-0.006$ \\
$\mu$ & 0.005 \\
\hline
\end{tabular}


HCs dropping probability, $E[V]$ are set to be 20 , 40 , or $60 \mathrm{~km} / \mathrm{hr}$. The arrival rate of NCs varies from 0.001 to 0.006 and the departure rate of NCs is fixed at 0.005 . The total number of MSs in the macro cell is 200 . Then, the corresponding transition matrix and the associated transition rates between any two states can be derived. All of the state probabilities are obtained by running the calculation on MATLAB. The Excel data for the analytical results are included within the supplementary information file and the performance metrics are presented in Figures 5-9.

\subsection{Results and Discussions}

Fixing the radius of a small cell $(r)$ at a medium size, 150 meters, Figures 5-9 show the performance measures derived in Section 3.3 versus different traffic loads. In Figure 5(a) and Figure 5(b), it is observed that the NC blocking probabilities in a sector and a small cell, $P b_{s r}$ and $P b_{s c}$ both increase with the system traffic load and the average moving speeds of MSs since the increment in traffic load or MS moving speed results in more NCs and HCs. Besides, it is found that the number of sectors ( $n$ ) has opposing influences on $P b_{s r}$ and $P b_{s c}$. Since the greater $n$ is, the less the sector region, $P b_{s r}$ increases with $n$ due to more channels being occupied by handoff calls. On the contrary, $P b_{s c}$ for $n=6$ is lower than that for $n=3$ because an even number of sectors (e.g., $n=6$ ) has more channels allocated for a small cell than an odd number of sectors (e.g., $n=3$ ). Figure 6 shows the HC dropping probabilities, $P b_{s \Sigma_{-} s n} P b_{s c_{-} s n}$ and $P b_{s r_{-} s c}$. From Figures 6(a)-(c), it is interesting that any one of the three HC dropping probabilities

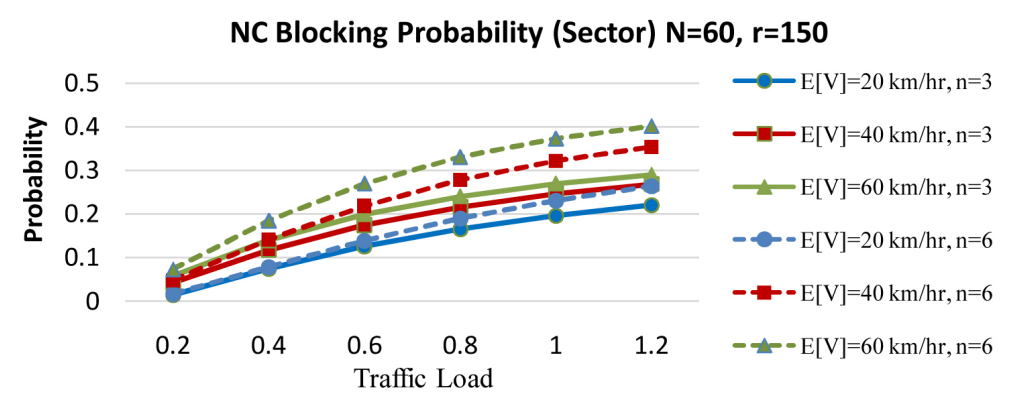

(a)

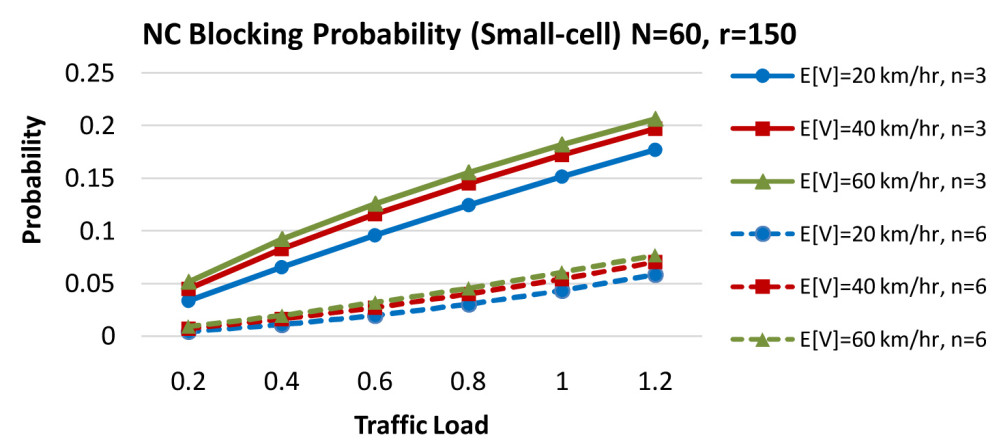

(b)

Figure 5. The NC blocking probability versus traffic load. (a) $P b_{s i}$; (b) $P b_{s c \text {. }}$ 


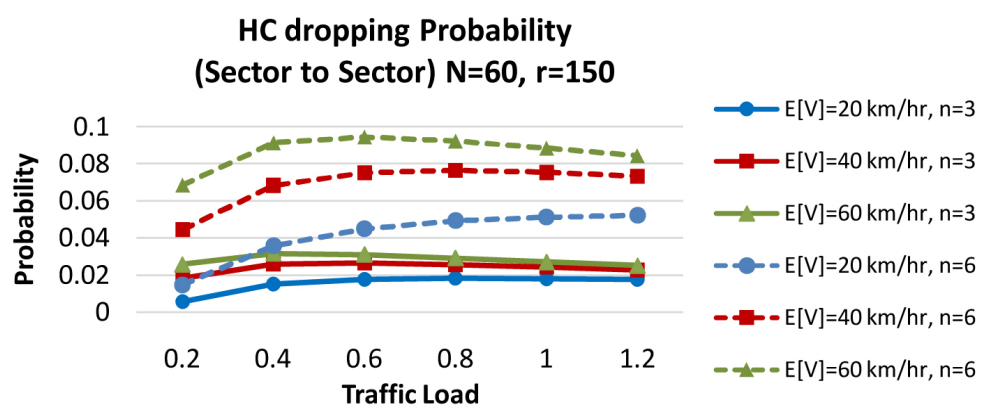

(a)

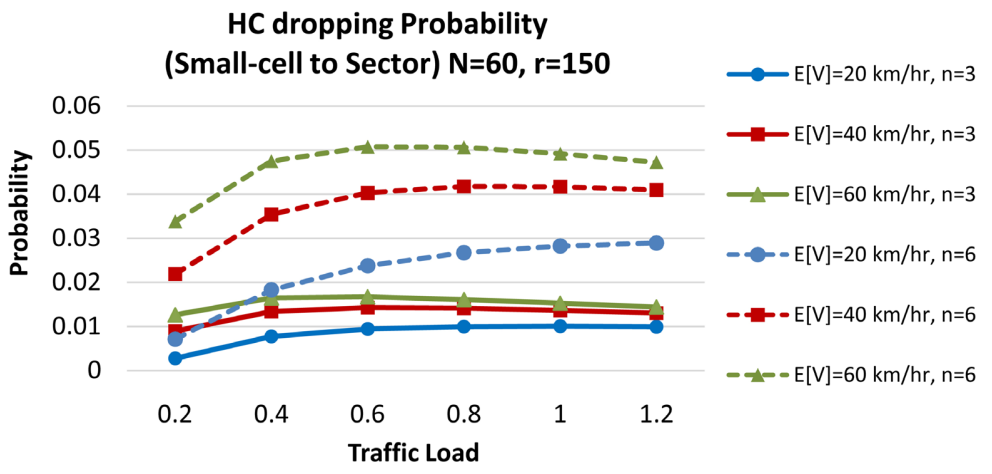

(b)

HC dropping Probability

(Sector to Small-cell) $\mathbf{N}=60, r=150 \quad \longrightarrow-E[V]=20 \mathrm{~km} / \mathrm{hr}, \mathrm{n}=3$

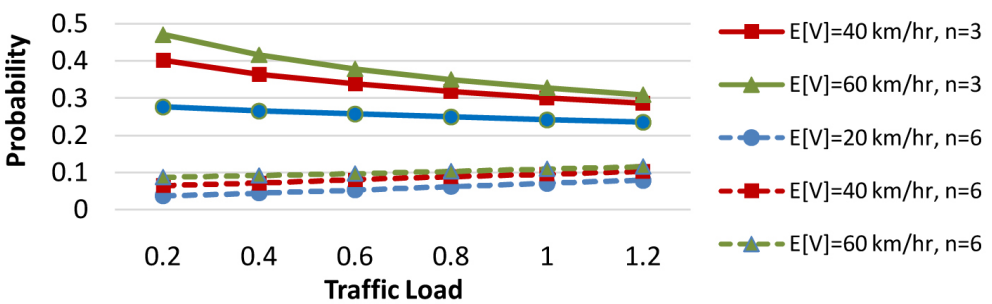

(c)

Figure 6. The HC dropping probability versus traffic load. (a) $P d_{s r_{-} s i} ;$ (b) $P d_{s c_{-} s r} ;$ (c) $P d_{s r_{-} s c}$.

always declines when the traffic load reaches a certain level. This is because larger traffic load means more NCs initiating in the system but the number of HCs remains the same and may get services more probably due to a higher percentage of NCs getting blocked.

To evaluate the influences of $r$ and $n$ on the AWT and NT, the average moving speed of MSs is fixed at $40 \mathrm{~km} / \mathrm{hr}$. Compared to Figure 5 and Figure 6, we can see from Figure 7 and Figure 8 that the same influence of the traffic load on the AWT of NCs and HCs for both a sector and a small cell. From Figure 7(a) and Figure 7(b), both $W n_{s r}$ and $W h_{s r}$ for $n=3$ are larger than those for $n=6$ because the system capacity enhanced by using FFR is more obvious when $n$ is even. Besides, no matter what $n$ is, $W n_{s r}$ increases but $W h_{s r}$ decreases with the increment in traffic load when $r$ becomes small. This is because smaller embedded-cell coverage makes a sector region larger and handoffs generating from an 


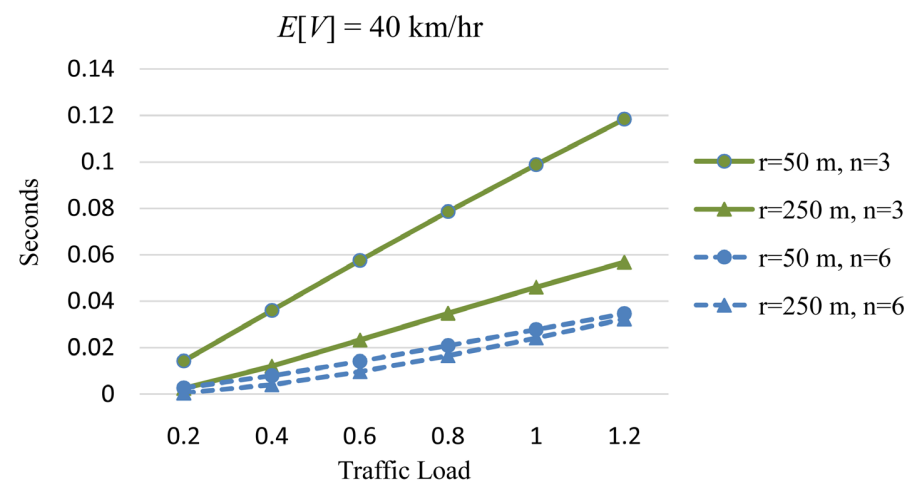

(a)

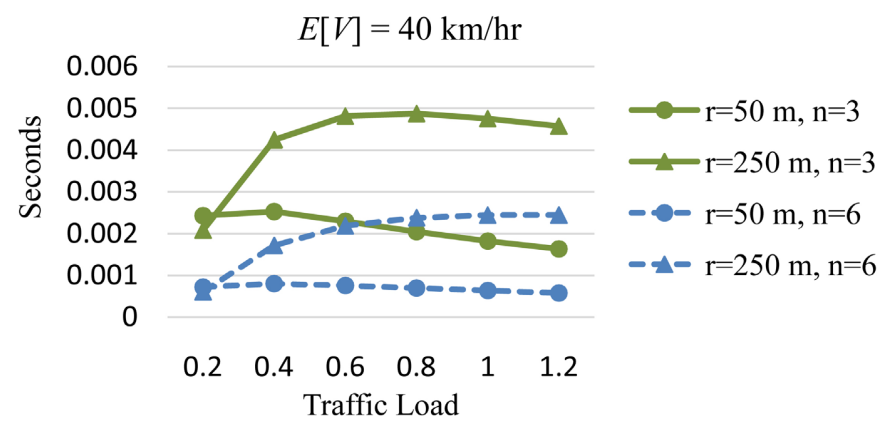

(b)

Figure 7. The average waiting time in a sector versus traffic load. (a) $W n_{s i}$; (b) $W h_{s r}$.

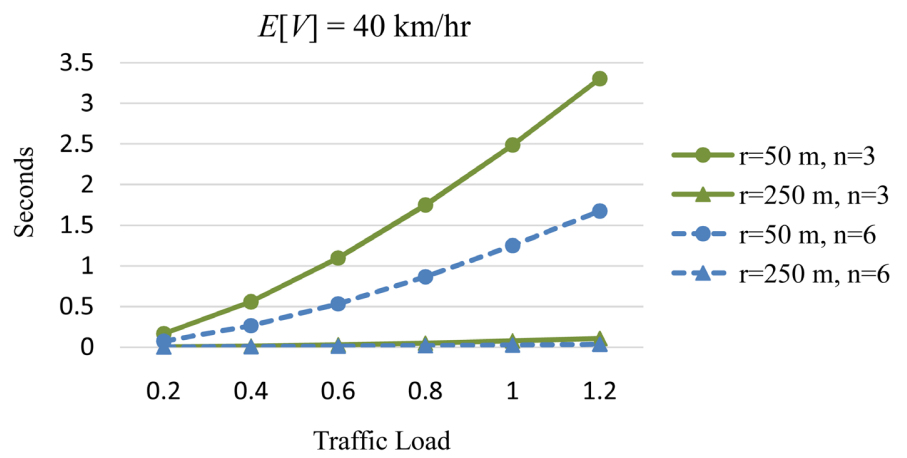

(a)

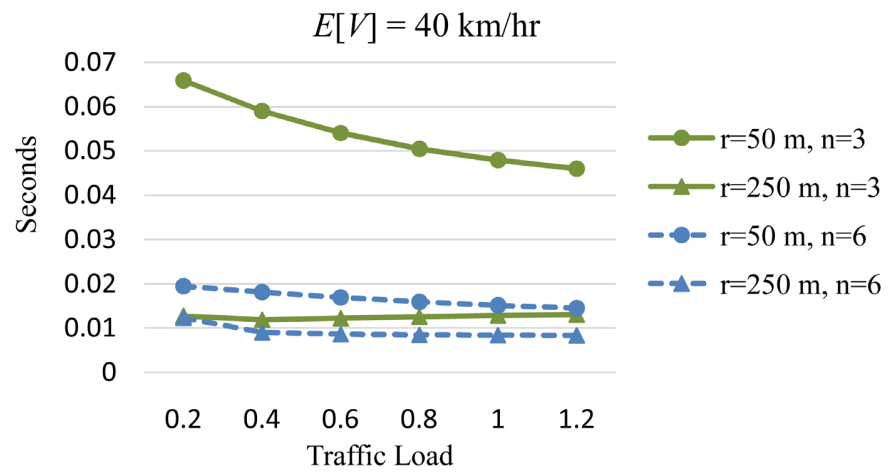

(b)

Figure 8. The average waiting time in a small cell versus traffic load. (a) $W n_{s c}$; (b) $W h_{s c}$. 


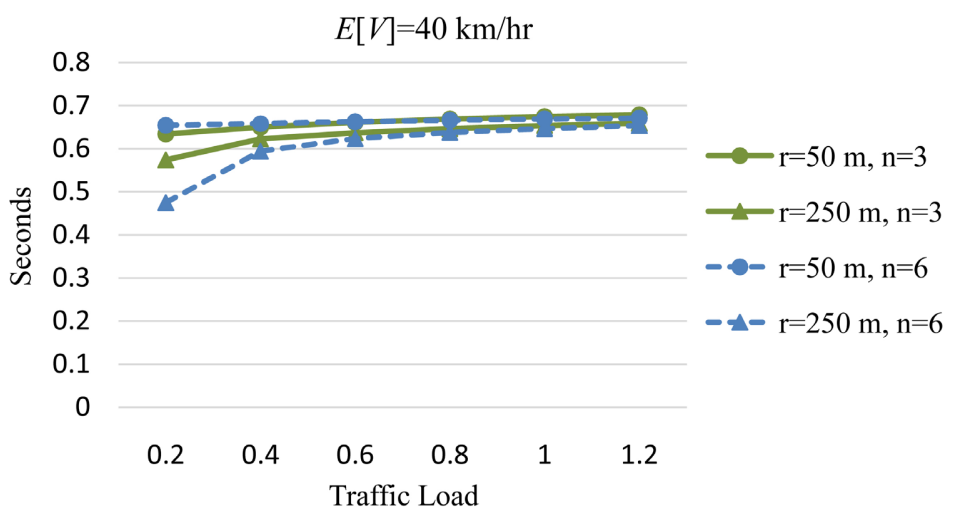

(a)

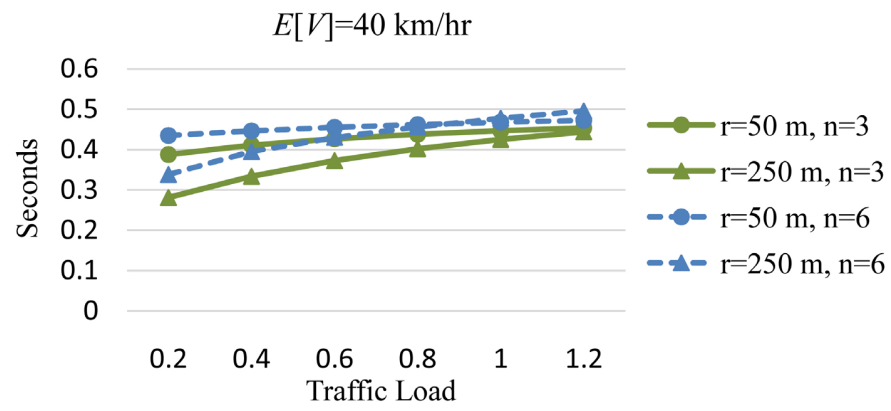

(b)

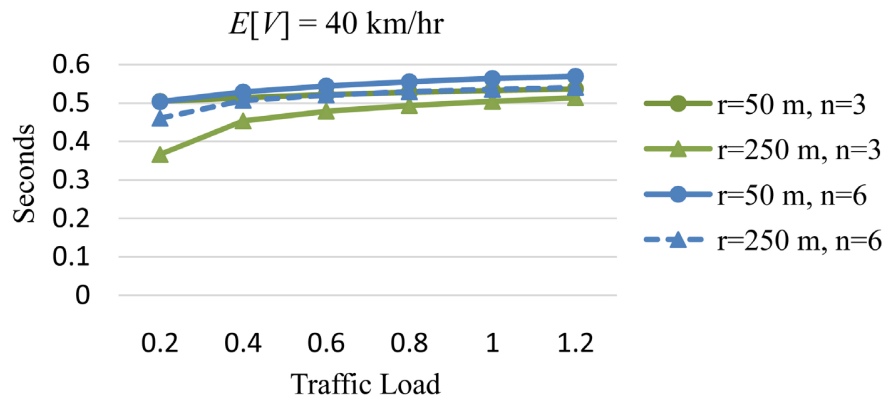

(c)

Figure 9. The normalized throughput versus traffic load. (a) $\Gamma_{s r}$; (b) $\Gamma_{s c}$; (c) $\Gamma_{m c}$.

embedded cell to a sector take place more frequently to get services in a sector region in which less of $N_{s r}$ are available and then, less of NCs can get services. Consequently, a decreasing number of NCs getting services brings about a decreasing number of HCs. On the other hand, as observed in Figure 8(a) and Figure 8(b), both $W n_{s c}$ and $W h_{s c}$ increase with the increment in traffic load when $r$ becomes small. This is simply because a smaller proportion of small cell coverage to sector region has less number of channels in a small cell.

As to the normalized throughput, we can see from Figure 9(a) and Figure 9(b) that both $\Gamma_{s r}$ and $\Gamma_{s c}$ are larger for $r=50$ than those for $r=250$, since a smaller size of an embedded cell results in a smaller $N_{s c}$ and more HCs generating from a small cell to its associated sector even though $N_{s r}$ is greater. At last, it is demonstrated from Figure 9(c) that using FFR can enhance the system capac- 
ity because the NT for the macro cell $\left(\Gamma_{m c}\right)$ for $n=6$ is larger than that for $n=3$ no matter what $r$ is.

\section{Conclusion}

We have presented a sectored cellular network with small cells (SCNSC) architecture. In the SCNSC, a multi-dimensional antenna is used for sectoring a macro cell and each sector is installed with a small cell to support the huge amounts of data transmitted through hot spots and to increase cell coverage in buildings. The overall system capacity is enhanced by using FFR. We have built an analytical model using Markov chain to do topological evaluation on the proposed SCNCS. The performance measures, including new-call blocking and handoffcall dropping probabilities, average waiting times, and normalized throughputs, are derived for a sector and its embedded small cell. From our analytical results, we have demonstrated that the new-call blocking and handoff-call dropping probabilities in a small cell can be decreased by increasing the number of sectors or the radius of a small cell. On the contrary, the handoff-call dropping probability in a sector can be decreased by decreasing the number of sectors or the radius of a small cell. To maximize the normalized throughput of the system, we can increase the number of sectors and decrease the proportion of small cell area to sector region. In summary, adjusting either the number of sectors or the coverage of an embedded cell can satisfy different service demands.

\section{Conflicts of Interest}

The authors declare no conflicts of interest regarding the publication of this paper.

\section{References}

[1] Chen, J., Ge, X. and Ni, Q. (2019) Coverage and Handoff Analysis of 5G Fractal Small Cell Networks. IEEE Transactions on Wireless Communications, 18, 12631276. https://doi.org/10.1109/TWC.2018.2890662

[2] Agiwal, M., Roy, A. and Saxena, N. (2016) Next Generation 5G Wireless Networks: A Comprehensive Survey. IEEE Communications Surveys \& Tutorials, 18, 1617-1655. https://doi.org/10.1109/COMST.2016.2532458

[3] Jiang, H., Xiao, Z., Li, Z., Xu, J., Zeng, F. and Wang, D. (2020) An Energy-Efficient Framework for Internet of Things Underlaying Heterogeneous Small Cell Networks. IEEE Transactions on Mobile Computing. https://doi.org/10.1109/TMC.2020.3005908

[4] Elhoushy, S. and Hamouda, W. (2020) Performance of Distributed Massive MIMO and Small-Cell Systems under Hardware and Channel Impairments. IEEE Transactions on Vehicular Technology, 69, 8627-8642. https://doi.org/10.1109/TVT.2020.2998405

[5] Paranthaman, V.V., Mapp, G., Shah, P., Nguyen, H.X. and Ghos, A. (2015) Exploring Markov Models for the Allocation of Resources for Proactive Handover in a Mobile Environment. 40th IEEE Local Computer Networks Conference Workshops, Clearwater Beach, 26-29 October 2015, 855-861. 
https://doi.org/10.1109/LCNW.2015.7365938

[6] Xu, J., Zhao, Y. and Zhu, X. (2014) Mobility Model Based Handover Algorithm in LTE-Advanced. 2014 10th International Conference on Natural Computation (ICNC), Xiamen, 19-21 August 2014, 230-234. https://doi.org/10.1109/ICNC.2014.6975840

[7] Bagdure, N. and Ambudkar, B. (2015) Reducing Delay during Vertical Handover. International Conference on Computing Communication Control and Automation, Pune, 26-27 February 2015, 200-204. https://doi.org/10.1109/ICCUBEA.2015.44

[8] Tseng, P.H., Feng, K.T. and Huang, C.H. (2014) POMDP-Based Cell Selection Schemes for Wireless Networks. IEEE Communications Letters, 18, 797-800.

https://doi.org/10.1109/LCOMM.2014.031414.132731

[9] Hossain, R., Real, A.H. and Rahman, A. (2016) Handover Management in Heterogeneous Network. 18th International Conference on Computer and Information Technology, Dhaka, 21-23 December 2015, 87-92. https://doi.org/10.1109/ICCITechn.2015.7488048

[10] Kim, S.Y., Ryu, S., Cho, C.H. and Lee, H.W. (2013) Performance Analysis of a Cellular Network Using Frequency Reuse Partitioning. International Journal on Performance Evaluation, 70, 77-89. https://doi.org/10.1016/j.peva.2012.09.006

[11] Chowdhury, M.Z., Jang, Y.M. and Haas, Z.J. (2013) Call Admission Control Based on Adaptive Bandwidth Allocation for Wireless Networks. Journal of Communications and Networks, 15, 15-24. https://doi.org/10.1109/JCN.2013.000005

[12] Fazio, P., Tropea, M. and Marano, S. (2013) A Distributed Hand-Over Management and Pattern Prediction Algorithm for Wireless Networks With Mobile Hosts. 9th International Wireless Communications and Mobile Computing Conference, Sardinia, 1-5 July 2013, 294-298. https://doi.org/10.1109/IWCMC.2013.6583575

[13] Sezginer, S. and Sari, H. (2009) Full Frequency Reuse in OFDMA-Based Wireless Networks with Sectored Cells. 2009 IEEE Wireless Communications and Networking Conference, Budapest, 5-8 April 2009, 1-4. https://doi.org/10.1109/WCNC.2009.4917732

[14] Ge, X., Han, T., Zhang, Y., Mao, G., Wang, C.X., Zhang, J., Yang, B. and Pan, S. (2014) Spectrum and Energy Efficiency Evaluation of Two-Tier Femtocell Networks with Partially Open Channels. IEEE Transactions on Vehicular Technology, 63, 1306-1319. https://doi.org/10.1109/TVT.2013.2292084

[15] Le, L.B., Niyato, D., Hossain, E., Kim, D.I. and Hoang, D.T. (2013) QoS-Aware and Energy-Efficient Resource Management in OFDMA Femtocells. IEEE Transactions on Wireless Communications, 12, 180-194. https://doi.org/10.1109/TWC.2012.120412.120141

[16] Le, L.B., Hossain, E., Niyato, D. and Kim, D.I. (2013) Mobility-aware Admission Control with QoS Guarantees in OFDMA Femtocell Networks. IEEE International Conference on Communications, Budapest, 9-13 June 2013, 2217-2222. https://doi.org/10.1109/ICC.2013.6654857

[17] Abonyi, D. and Rigelsford, D. (2018) A System for Optimizing Small-Cell Deployment in 2-Tier HetNets. IEEE 23 rd International Workshop on Computer Aided Modeling and Design of Communication Links and Networks, Barcelona, 17-19 September 2018, 1-6. https://doi.org/10.1109/CAMAD.2018.8514981

[18] Chen, J.S., Chen, H.-C., Huang, Y.-F., Wei, C.-C. and Chuang, K.-C. (2012) An Adaptive Capacity Enhancement Strategy for Sector-Based Cellular Systems. International Journal on Computers and Mathematics with Applications, 64, 1462-1472. https://doi.org/10.1016/j.camwa.2012.03.093

[19] Sheu, T.L., Lin, B.J. and Ma, C.G. (2014) Call Blocking and Dropping in Sectorized 
Cellular Networks with Fractional Frequency Reuse. Proceedings of World Telecommunications Congress, Berlin, 1-3 June 2014, 1-6.

[20] Martinez, J.L., Solana, A.H. and Valdovinos, A. (2012) On the Dependence between FPC and ICIC in SC-FDMA Cellular Systems. 2012 IEEE Vehicular Technology Conference-Fall, Quebec, 3-6 September 2012, 1-5.

[21] He, J., Tang, Z., Ding, Z. and Wu, D. (2018) Successive Interference Cancellation and Fractional Frequency Reuse for LTE Uplink Communications. IEEE Transactions on Vehicular Technology, 67, 10528-10542. https://doi.org/10.1109/TVT.2018.2865814

[22] Wu, J., Chu, X., López-Pérez, D. and Wang, H. (2012) Femtocell Exclusion Regions in Hierarchical 3-Sector Macrocells for Co-Channel Deployments. 1st IEEE International Conference on Communications in China, Beijing, 15-17 August 2012, 541 545. https://doi.org/10.1109/ICCChina.2012.6356943

[23] Wu, J., Chu, X. and Perez, D.L. (2012) Downlink Outage Probability of Co-Channel Femtocells in Hierarchical 3-Sector Macrocells. IEEE Communications Letters, 16, 698-701. https://doi.org/10.1109/LCOMM.2012.030912.120235

[24] Kalbkhani, H., Shayesteh, M.G., Jafarpour-Alamdari, S. and Solouk, V. (2012) Outage Probability of Femtocells in Two-Tier Networks with 6-Sector Macrocells. 6th International Symposium on Telecommunications, Tehran, 6-8 November 2012, 283 288. https://doi.org/10.1109/ISTEL.2012.6482998

[25] Tsai, A.-H. (2018) Two-Tier Interference Mitigation with Directional Antennas for Small-Cells in an Apartment Building. 15th International Symposium on Wireless Communication Systems, Lisbon, 28-31 August 2018, 1-5. https://doi.org/10.1109/ISWCS.2018.8491195

[26] Samal, C., Samal, L. and Das, S. (2014) By Fractional Frequency Reuse Mitigate Interference in Heterogeneous Femto and Macro Cell Networks. 2014 International Conference on Green Computing Communication and Electrical Engineering, Coimbatore, 6-8 March 2014, 1-5. https://doi.org/10.1109/ICGCCEE.2014.6922392

[27] López-Pérez, D., Claussen, H. and Ho, L. (2015) The Sector Offset Configuration Concept and Its Applicability to Heterogeneous Cellular Networks. IEEE Communications Magazine, 53, 190-198. https://doi.org/10.1109/MCOM.2015.7060504

[28] Xie, H. and Kuek, S. (1993) Priority Handoff Analysis. 43rd IEEE Vehicular Technology Conference, Secaucus, 18-20 May 1993, 855-858. https://doi.org/10.1109/VETEC.1993.510945

[29] Orsino, A., Guo, W. and Araniti, G. (2018) 5G Multiscale Mobility: A Look at Current and Upcoming Models in the Next Technology Era. IEEE Vehicular Technology Magazine, 13, 120-129. https://doi.org/10.1109/MVT.2017.2772646

[30] Ross, S.M. (2000) Introduction to Probability Models, 7th Edition, Academic Press, San Diego. 